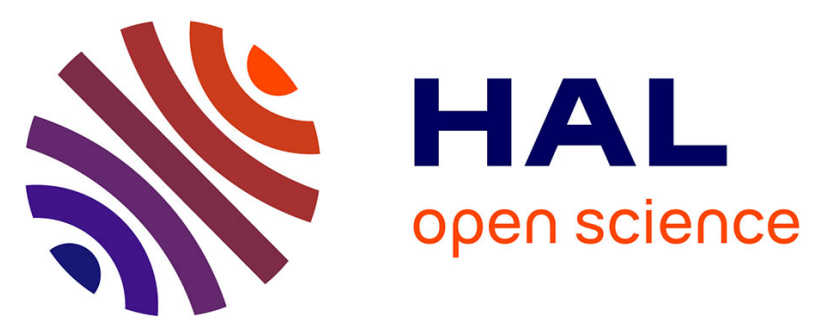

\title{
How much could a microbicide's STI-efficacy contribute to reducing HIV-risk and the level of condom use needed to lower risk? Model estimates
}

Anna Mary Foss, Peter Thomas Vickerman, Michel Alary, Charlotte Helen Watts

\section{To cite this version:}

Anna Mary Foss, Peter Thomas Vickerman, Michel Alary, Charlotte Helen Watts. How much could a microbicide's STI-efficacy contribute to reducing HIV-risk and the level of condom use needed to lower risk? Model estimates. Sexually Transmitted Infections, 2009, 85 (4), pp.276. 10.1136/sti.2008.032458. hal-00552793

\section{HAL Id: hal-00552793 \\ https://hal.science/hal-00552793}

Submitted on 6 Jan 2011

HAL is a multi-disciplinary open access archive for the deposit and dissemination of scientific research documents, whether they are published or not. The documents may come from teaching and research institutions in France or abroad, or from public or private research centers.
L'archive ouverte pluridisciplinaire HAL, est destinée au dépôt et à la diffusion de documents scientifiques de niveau recherche, publiés ou non, émanant des établissements d'enseignement et de recherche français ou étrangers, des laboratoires publics ou privés. 


\section{FULL TITLE:}

\section{How much could a microbicide's STI-efficacy contribute to reducing HIV-risk and the level of condom use needed to lower risk? Model estimates}

\section{SHORT TITLE:}

\section{Importance of STI-efficacy for microbicides}

Anna M Foss ${ }^{1}$, Peter T Vickerman ${ }^{1}$, Michel Alary ${ }^{2}$, and Charlotte H Watts ${ }^{1}$

${ }^{1}$ London School of Hygiene \& Tropical Medicine, Keppel Street, London WC1E 7HT,

UK; ${ }^{2}$ Centre hospitalier affilié universitaire de Québec, Québec, Canada.

For correspondence and reprint requests please contact:

Anna Foss

Department of Public Health \& Policy

London School of Hygiene \& Tropical Medicine

Keppel Street

London WC1E 7HT

UK 
Email: anna.foss@1shtm.ac.uk

Tel: +44 (0)20 76127891

Fax: +44 (0)20 76375391

Abstract word count: 247

Text word count: 2,854

Number of Tables: 2

Number of Figures: 2

The Corresponding Author has the right to grant on behalf of all authors and does grant on behalf of all authors, an exclusive licence (or non exclusive for government employees) on a worldwide basis to the BMJ Publishing Group Ltd to permit this article (if accepted) to be published in STI and any other BMJPGL products and sub-licences such use and exploit all subsidiary rights, as set out in our licence http://sti.bmijournals.com/ifora/licence.pdf). 


\section{AUTHOR CONTRIBUTIONS}

Anna Foss (AF) took the lead in the analysis and made substantial contributions to the conception, design of the analysis and interpretation of the results presented. Peter Vickerman (PV) and Charlotte Watts (CW) contributed to the conception and design of the analysis, and helped to interpret the findings. PV and CW provided substantial input into several drafts of the paper. Michel Alary (MA) helped with interpreting the data from Cotonou and commented on drafts of the paper.

\section{KEY MESSAGES}

1. A microbicide's STI-efficacy may have a substantial impact on STI and HIV incidence among high-risk groups.

2. For highly transmissible STIs, such as gonorrhoea, as the STI prevalence increases the relative reduction in HIV-risk increases at first, due to the proportion of HIV-risk attributable to the STI increasing, but then decreases at higher prevalences as the STI becomes harder to control.

3. For a less transmissible STI, like chlamydia, the STI can still be impacted upon with a moderate/high STI-efficacious microbicide even at high STI prevalences.

4. The variation in the 'condom migration thresholds' (the reduction in condom use following microbicide introduction that can be tolerated without increasing HIVrisk), for different STI-efficacies and STI prevalences, may be difficult to accurately measure. 


\section{ABSTRACT}

\section{Objective}

This study explores the potential contribution of a microbicide's STI-efficacy in reducing a female sex worker's (FSW's) risk of STI and HIV infection. The study then investigates whether the threshold, for the reduction in condom use following microbicide introduction that can be tolerated without increasing HIV-risk, is affected by the STI-efficacy.

\section{Methods}

A dynamic model describing the transmission of a bacterial STI between FSWs and their clients was coupled with a static HIV model. The model uses data from Cotonou, Benin (1998-99), for illustration, to estimate the change in risk following the introduction of $50 \%$ HIV-efficacious microbicides of different STI-efficacies, used in 50\% of sex acts when a condom is not used. The condom migration thresholds were estimated. The degree to which the findings are influenced by STI prevalence was explored.

\section{Results}

For highly transmissible STIs, there is a non-monotonic relationship between STI prevalence and microbicide impact on HIV with the relative reduction in HIV-risk first increasing, due to the proportion of HIV-risk attributable to the STI increasing, but then decreasing at high prevalences as the STI becomes harder to control. A less transmissible STI can still be impacted upon with a moderate/high STI-efficacy microbicide even at high STI prevalences. This relationship is also reflected in the condom migration thresholds. 


\section{Conclusions}

A microbicide's STI-efficacy may have a substantial impact on STI and HIV incidence among high-risk groups. The variation in the condom migration thresholds for different STI-efficacies and STI prevalences may be difficult to accurately measure.

Key words: HIV, sexually transmitted infections, vaginal microbicides, condoms, mathematical models 


\section{INTRODUCTION}

A microbicide is a substance capable of reducing the transmission of HIV and other sexually transmitted infections (STIs). Studies have shown that many promising candidates prevent infection in the laboratory, and three are in large-scale HIVeffectiveness clinical trials. ${ }^{1}$

At present it is unknown what HIV- and STI-efficacy we can expect microbicides to have, where 'efficacy' is defined here as the percentage reduction in the transmission probability per sex act. Two of the products currently in late stage clinical HIVeffectiveness trials have demonstrated STI-efficacy, in-vitro or in animal models, including gonorrhoea, herpes simplex virus and chlamydia, although some products in

development have no STI-efficacy. ${ }^{1-3}$ Mathematical modelling suggests that a microbicide's STI-efficacy could play an important role in HIV transmission, particularly in settings where prevalence is relatively low/moderate, and can be impacted upon. ${ }^{4}$ However, it is not possible to infer from this analysis how important a microbicide's STIefficacy may be in other epidemiological settings, beyond the two African settings considered.

This paper uses modelling to explore the potential contribution of a microbicide's STIefficacy in reducing a female sex worker's (FSW's) risk of STI and HIV infection for a range of different STI prevalence and efficacy assumptions. Since there is concern that the introduction of microbicides may result in women who are using condoms switching 
to less effective microbicides (termed 'condom migration'), ${ }^{5}$ the implications of these results for the degree to which condom migration can be tolerated without increasing HIV-risk are explored. The findings are compared with a previous analysis that also estimated the 'condom migration threshold', but assumed that microbicides have equal HIV- and STI-efficacy. ${ }^{6}$ 


\section{METHODS}

Foss et al (2003) presented a standard Bernoulli equation describing an individual's risk of becoming HIV infected over a fixed time-period, referred to as the 'static HIV model' (Appendix). ${ }^{6}$ An analogous equation can also be used to describe the probability of STI acquisition $\left(\pi_{\mathrm{s}}\right)$ (Appendix). In both cases, the main assumption is that the probability of transmission per sex act is independent of previous sex acts.

\section{Model adaptations}

To investigate the role of microbicide STI-efficacy in HIV-risk, the static model was revised by coupling it with a simple dynamic STI model, to reflect possible changes in STI prevalence. This was required so that the benefit of any STI-efficacy of the microbicide, and any potential negative impact of condom migration, are incorporated as effects on STI transmission.

The model simulated the transmission of a curable STI between FSWs and their male clients, and their risk of acquiring HIV. In order to represent, in a simple way, infection from those beyond the closed intervention populations modelled, a seeding term was included in the model equations for males. Since microbicides would be targeted only to FSWs in contact with an intervention, this seed represents infections from others who do not have access to microbicides. The seed acts as a sustaining force so that the STI is not eradicated, but contributes little to the overall client prevalence $(0.8 \%)$. 
The dynamic STI model was used to estimate the equilibrium STI prevalence before and after the introduction of microbicides, assuming that HIV does not affect the equilibrium STI prevalence over a short timeframe. Numerical methods were used to approximate the change in STI prevalence over time. For this, a dynamic deterministic compartmental model of the transmission of a curable STI was built in Microsoft $®$ Excel (Appendix).

\section{Parameterisation and microbicide efficacy scenarios}

As in Foss et al (2003), ${ }^{6}$ data collected in a cross-sectional survey (1998-99) from Cotonou, Benin, is used as an illustrative case-example, to reflect a setting with an ongoing intervention that, at this time, had achieved moderate levels of condom use during commercial sex. Four scenarios are considered for the STI-efficacy of the microbicide: $0 \%, 25 \%, 50 \%$ and $75 \%$. For illustration, microbicides are assumed to have $50 \%$ HIV-efficacy and be used in $50 \%$ of non-condom-protected sex acts, to reflect the statistical power of the microbicide efficacy trials and using data from the spermicide trials on levels of use. ${ }^{67}$ It is assumed that if a microbicide is used with a condom in a sex act then the additional protection offered by the microbicide is negligible compared to that provided by the condom. Using the input parameters listed in Table 1, the STI model simulated an epidemic, to reach equilibrium STI prevalence values, giving an indication of how changes in condom and microbicide use affect STI prevalence.

[INSERT Table 1] 


\section{Model fitting and STI prevalence scenarios}

The model was fit to data on the prevalence of gonorrhoea among FSWs and clients collected in the 1998-99 survey in Cotonou. Gonorrhoea prevalence values were used since this was the most prevalent STI in Cotonou for which there were available data for the groups considered. ${ }^{8101128}$ The fitting procedure involved using the 'Solver' add-in to Microsoft ${ }^{\circledR}$ Excel to fit to these STI prevalences by adjusting specific parameters, namely the duration of the STI, the STI transmission probabilities, and the sustaining seed. These parameters were varied within ranges based on data from the literature for the duration of gonorrhoea ${ }^{25}$ (incorporating the effects of treatment ${ }^{26}$, see Table 1) and its

$\operatorname{transmissibility~}^{15}{ }^{16}$, and assuming that the sustaining seed for the STI was $\leq 1 \%$ (Appendix).

The values used and obtained through the fitting procedure, along with the equilibrium STI prevalence estimates before and after microbicide introduction, were then used in the static HIV model. The HIV model incorporates the facilitating effect of the STI on HIV transmission. $^{2324}$

Using data on the number of clients reported by FSWs in Cotonou (1998-99) gave the 'medium' STI prevalence scenario (21.0\% among FSWs and 5.4\% among clients). In order to consider the generalisability of the findings to other times and settings, the number of clients per FSW per month was adjusted to generate a reasonable range of STI prevalences. Within this range, specific 'low' and 'high' curable STI prevalence scenarios were obtained by, respectively, halving and doubling the number of clients per 
FSW per month. The 'low' and 'high' STI prevalence scenarios obtained for FSWs (and their clients) were $5.5 \%(2.1 \%)$, and $38.6 \%(8.8 \%)$, respectively.

\section{Steps of analysis}

In the analysis, the revised model (dynamic STI-static HIV coupled model) is first used to explore to what degree a microbicide's STI-efficacy can reduce an individual's risk of STI and HIV infection per month. Then, allowing for condom migration, this model is used to compare the 'condom migration thresholds' for microbicides of different STIefficacies, introduced into settings with various STI prevalence profiles.

A sensitivity analysis was also conducted to explore the effect on the findings of increasing from 2 to 6 the multiplicative STI cofactor facilitating HIV transmission per sex act. In order to investigate the generalisability of the findings to other curable STIs, such as chlamydia in other settings, ${ }^{29-31}$ an additional model refit to the same prevalences was identified manually by assuming a longer duration of the STI and lower transmissibility, ${ }^{1718252732}$ and the analysis was repeated using this refit. The duration of the STI was then varied, rather than the number of clients per FSW, as an alternative method of exploring the same range of STI prevalences with this refit, to reflect different levels of STI treatment. 


\section{RESULTS}

\section{Reduction in STI-risk}

Assuming that the consistency of condom use remains at $56 \%$, in low STI prevalence settings, it is relatively easy to have an impact on the STI with a reasonably low or medium STI-efficacy microbicide that is used in $50 \%$ of non-condom-protected sex acts, and so increasing STI-efficacy further has limited added value. In higher STI prevalence settings it is harder to control the STI since the risk of infection and re-infection is also high, and so the incremental benefit increases steadily as STI-efficacy increases.

\section{Reduction in HIV-risk}

For the 'medium' STI prevalence scenario (21\% among FSWs), a zero HIV-efficacy microbicide with $50 \%$ STI-efficacy reduces HIV-risk by $11 \%$, while a $50 \%$ HIV-efficacy microbicide with zero STI-efficacy reduces risk by $23 \%$. So a microbicide that is only $50 \%$ efficacious against STI (with no HIV-efficacy) may still have $47 \%$ of the HIVimpact that a 50\% HIV-efficacious microbicide has without STI-efficacy.

Figure 1 illustrates the reduction in HIV-risk from a 50\% HIV-efficacious microbicide (for various STI-efficacies), introduced into a range of STI prevalences, with condomconsistency remaining at 56\%, and with other parameters as in Table 1.

[INSERT Figure 1] 
In the original model fit (Figure 1a), the potential relative reduction in HIV-risk among FSWs for a 50\% HIV-efficacious microbicide with 50\% STI-efficacy varies from 25$33 \%$ depending on the STI prevalence. There is a non-monotonic relationship between STI prevalence and microbicide impact, with the relative reduction in HIV-risk first increasing as the STI prevalence increases, due to the proportion of HIV-risk attributable to the STI increasing, but then decreasing at higher prevalences as the STI becomes harder to control.

The same pattern is observed if the multiplicative STI cofactor facilitating HIV transmission per sex act is increased from 2 to 6 , but the reduction in HIV-risk is greater for each STI-efficacy scenario and there is more variation across the STI prevalences. For a $50 \%$ HIV-efficacious microbicide with 50\% STI-efficacy the potential relative reduction in HIV-risk varies from $28-47 \%$, depending on the STI prevalence, if an STI cofactor of 6 is used.

If the model is refit assuming a longer duration of the STI and lower transmissibility, the potential relative reduction in HIV-risk is shown in Figure 1b. If the duration of the STI in the refit is adjusted to vary the STI prevalence across the same range as in Figure 1a, the non-monotonic relationship is less pronounced in the $0 \%$ and $25 \%$ STI-efficacy scenarios and the relationship is actually monotonic for the $50 \%$ and $75 \%$ STI-efficacy scenarios in this STI prevalence range. This difference between Figure 1a and Figure 1b is due to the different STI transmissibilities assumed in each. In Figure 1a the STI is 
highly transmissible, meaning it is hard to control the STI when the STI prevalence is high, resulting in the microbicide achieving less impact on HIV-risk, while in Figure $1 \mathrm{~b}$ the STI is half as transmissible and this means that the STI can be controlled, even at high prevalences, if the microbicide's efficacy is $50 \%$ or more.

\section{Condom migration thresholds}

Based on the parameters in Table 1, where the condom consistency was $56 \%$ before microbicide introduction, Table 2 shows that more condom migration can be tolerated as the microbicide STI-efficacy is increased.

In the original model fit, the thresholds are more sensitive to changes in the microbicide's STI-efficacy (0-75\%) in the medium STI scenario (10-20\% with STI cofactor of 2 or 6$23 \%$ with STI cofactor of 6), than in the 'low' or 'high' STI prevalence scenarios. The values in Table 2 change by at most $1 \%$ for the refit compared to the original fit.

\section{[INSERT Table 2]}

The thresholds are also fairly similar across the different STI prevalence scenarios. The small differences are due to the interplay between increasing STI prevalence acting to increase the proportion of HIV-risk attributable to the STI while also potentially making it harder to control the STI, particularly if it is highly transmissible, as observed in Figure 1. At the threshold level of $16 \%$ condom migration for a $50 \% \mathrm{HIV}$ - and $50 \%$ STIefficacious microbicide, a higher STI-efficacy microbicide will reduce HIV-risk and a 
lower STI-efficacy microbicide will increase HIV-risk. The magnitude of this decrease or increase in HIV-risk corresponds to the degree to which any more or less condom migration can be tolerated without increasing risk (Figure 2).

[INSERT Figure 2] 


\section{DISCUSSION}

The findings build on previous modelling that have either explored the importance of STI-efficacy for the HIV-impact of a microbicide in two settings, ${ }^{4}$ or have estimated the degree of condom migration that can occur without increasing risk following microbicide introduction. ${ }^{633} 34$ The modelling presented here extends the former analysis because it gives projections for a wide range of STI prevalences and STI-efficacies, and extends the latter analysis by exploring the effect of a microbicide's STI-efficacy on the migration thresholds.

Even fairly low STI-efficacy microbicides can have a large effect on the STI in settings with a low prevalence of curable STI. For highly transmissible STIs, such as gonorrhoea, there is a non-monotonic relationship between STI prevalence and microbicide impact on HIV with the relative reduction in HIV-risk first increasing, due to the proportion of HIV-risk attributable to the STI increasing, but then decreasing at higher prevalences as the STI becomes harder to control. For a less transmissible STI, like chlamydia, the STI can still be impacted upon with a moderate/high STI-efficacious microbicide even at high STI prevalences. This implies that, if the introduction of the microbicide leads to a change in the STI prevalence, the HIV-impact of the same microbicide will likely vary by setting. Additionally, the previous static HIV model estimates of the microbicide's impact on HIV-risk will be conservative in this case. 
Increasing the STI-efficacy of a microbicide from zero to $75 \%$ also increases the amount of condom migration that can be tolerated without increasing HIV-risk, but only by as much as 10 percentage points if the STI increases 2-fold the probability of HIV transmission per sex act. It may be hard to measure condom use differences to this degree of accuracy.

\section{Limitations}

As in the earlier work, using a static HIV model, even when coupled with a dynamic STI model, means is it not possible to estimate how a microbicide may impact on the HIV epidemic over longer time-periods. However, coupling a dynamic STI model with a static HIV model was a simpler intermediate step, using an appropriate level of complexity to address the questions posed, and has provided several key insights.

More recent data from Cotonou (2005) suggest that condom use during commercial sex has increased, and gonorrhoea and HIV prevalences have decreased substantially. ${ }^{35}{ }^{36}$ As a result, the present-day situation in Cotonou may more closely resemble the low STI prevalences presented here (in Figure 1) than the 'medium' STI prevalence scenario. At the new very high level of reported condom use (95\% at last sex), little if any condom migration can be tolerated without increasing HIV-risk. The findings from considering moderate and high STI prevalences have particular relevance to FSW populations in which moderate or high prevalences of curable STI remain, such as in the Philippines ${ }^{29}$, Vietnam ${ }^{37}$ and China ${ }^{3031} 38$ 
Only commercial sex was modelled since these were the partnerships of concern in terms of condom migration in the previous static modelling, ${ }^{6}$ and analysis of long-term partnerships in the general population concluded that the estimates from the static model were insensitive to variations in STI-efficacy explored using the revised model. ${ }^{39}$ Dynamic population-level HIV/STI modelling has been used to investigate this further, with data from Benin and South Africa. ${ }^{4}$ Using a slightly different parameterisation and scenarios from this analysis (in order to fit the more complex model), they agree with our finding that the sensitivity of a microbicide's HIV-impact to increases in STI-efficacy may reduce at very high STI prevalences.

Desai et al (2006) predicted that the fraction of HIV infections prevented in circumcised males that were attributable to STI was unlikely to be more than $10-20 \%$ in a population of low- and high-risk groups. ${ }^{40}$ This is lower than what is predicted here for a $50 \%$ HIVand STI- efficacious microbicide used in commercial sex, where up to $28 \%$ of the relative reduction in HIV-risk among FSWs was attributed to its STI-efficacy. However, for longterm partnerships in Cotonou, our modelling suggests that less than $10 \%$ of a microbicide's HIV-impact can be attributed to the microbicide's STI-efficacy. Additionally, unlike Desai et al (2006), who assumed male circumcision offered no direct protection to females, microbicides are here assumed to provide the same degree of protection to both males and females, which may not be the case. Currently, the phase 3 trials are focusing on measuring the effectiveness of microbicides in reducing the risk of HIV transmission from males to females only. ${ }^{141}$ Recent modelling work has investigated this issue of uni- versus bi-directional protection in exploring the potential contribution of 
a microbicide's STI-efficacy on the reduction in HIV incidence (effectiveness) observed in a phase 3 trial. $^{7}$ The difference in the reduction in HIV incidence in a trial or HIV-risk among women from a bi-directional versus a uni-directional microbicide may be fairly small initially, since any change in the HIV prevalence among males is likely only to be observable over a longer timeframe.

\section{Conclusions}

The findings illustrate that a microbicide's STI-efficacy may have a substantial impact on the incidence of HIV and curable STIs among high-risk groups, particularly in settings where the STI is relatively easy to control and a substantial proportion of HIV-risk is attributable to the STI. Although incorporating microbicide STI-efficacy into the model provided improved estimates of the migration threshold for FSWs, whether this adds important additional policy insights depends on whether the differences between the thresholds for different STI-efficacies are large enough to be measurable.

The analysis presented here highlights the importance of developing and evaluating microbicides with STI-efficacy, and so supports the Microbicide Development Programme trial having some STIs as secondary outcomes. Combination products, such as those based on antiretrovirals added into microbicides with STI-efficacy, should also be pursued. 


\section{ACKNOWLEDGEMENTS}

The microbicide work is funded by the Global Campaign for Microbicides at the Program for Appropriate Technology in Health (PATH), via a grant from USAID, and by the UK Department for International Development (DFID) through the Microbicide Development Programme, and the Research Programme Consortium for Research and Capacity Building in Sexual and Reproductive Health and HIV in Developing Countries. Anna Foss, Peter Vickerman and Charlotte Watts were also members of the Health Economics and Financing Programme and the Knowledge Programme on HIV/AIDS and STI, which were funded by DFID. Michel Alary is a National Researcher of the Fonds de la recherché en santé du Québec (grant \# 8722). We would also like to thank Lori Heise, Sevgi Aral and Geoff Garnett for their useful comments on this work. 


\section{REFERENCES}

1. Global Campaign for Microbicides, Alliance for Microbicide Development. Product Pipeline. September 2008. http://www.global-campaign.org/clientfiles/FS14AllianceProductPipeline[E]Sept08.pdf (accessed 10 September 2008).

2. Zeitlin L, Whaley KJ. Microbicides for preventing transmission of genital herpes. Herpes 2002;9(1):4-9.

3. De Clercq E. Clinical potential of the acyclic nucleoside phosphonates cidofovir, adefovir, and tenofovir in treatment of DNA virus and retrovirus infections. Clin Microbiol Rev 2003;16(4):569-96.

4. Vickerman P, Watts C, Delany S, et al. The importance of context: model projections on how microbicide impact could be affected by the underlying epidemiologic and behavioral situation in 2 African settings. Sex Transm Dis 2006;33(6):397-405.

5. Heise L. Topical microbicides: new hope for STI/HIV prevention: Center for Health and Gender Equity (CHANGE) 1999. http://www.genderhealth.org/.

6. Foss AM, Vickerman PT, Heise L, et al. Shifts in condom use following microbicide introduction: should we be concerned? AIDS 2003;17(8):1227-37.

7. Vickerman P, Foss A, Watts C. Using modeling to explore the degree to which a microbicide's sexually transmitted infection efficacy may contribute to the HIV effectiveness measured in phase 3 microbicide trials. J Acquir Immune Defic Syndr 2008;48(4):460-7.

8. Lowndes CM, Alary M, Gnintoungbe CA, et al. Management of sexually transmitted diseases and HIV prevention in men at high risk: targeting clients and nonpaying sexual partners of female sex workers in Benin. Aids 2000;14(16):2523-34. 9. Pinkerton SD, Abramson PR. Effectiveness of condoms in preventing HIV transmission. Social Science and Medicine 1997;44(9):1303-12.

10. Alary M, Mukenge-Tshibaka L, Bernier F, et al. Decline in the prevalence of HIV and sexually transmitted diseases among female sex workers in Cotonou, Benin, 19931999. Aids 2002;16(3):463-470.

11. Lowndes CM, Alary M, Meda $\mathrm{H}$, et al. Role of core and bridging groups in the transmission dynamics of HIV and STIs in Cotonou, Benin, West Africa. Sex Transm Infect 2002;78 Suppl 1:i69-77.

12. Royce RA, Sena A, Cates W, Jr., et al. Sexual transmission of HIV. N Engl J Med 1997;336(15):1072-8.

13. Boily MC, Baggaley RF, Wang L, et al. The risk of HIV-1 infection per sexual contact in absence of antiretroviral therapy: a systematic review and meta-analysis of observational studies. 17th International Society for Sexually Transmitted Diseases Research; 29 July - 1 August 2007; Seattle, Washington, USA [Abstract O-058]. http://www.isstdr.org/index.php?id=97.

14. Vickerman P, Watts C, Alary M, et al. Sensitivity requirements for the point of care diagnosis of Chlamydia trachomatis and Neisseria gonorrhoeae in women. Sex

Transm Infect 2003;79(5):363-7. 
15. Holmes KK, Johnson DW, Trostle HJ. An estimate of the risk of men acquiring gonorrhea by sexual contact with infected females. Am J Epidemiol 1970;91(2):170-4.

16. Hooper RR, Reynolds GH, Jones OG, et al. Cohort study of venereal disease. 1: The risk of gonorrhoea transmission from infected women to men. American journal of Epidemiology 1978;108(2):136-144.

17. Kretzschmar M, Welte R, van den Hoek A, et al. Comparative model-based analysis of screening programs for Chlamydia trachomatis infections. Am J Epidemiol 2001;153(1):90-101.

18. Lycke E, Lowhagen GB, Hallhagen G, et al. The risk of transmission of genital Chlamydia trachomatis infection is less than that of genital Neisseria gonorrhoeae infection. Sex Transm Dis 1980;7(1):6-10.

19. Quinn TC, Wawer MJ, Sewankambo N, et al. Viral load and heterosexual transmission of human immunodeficiency virus type 1. Rakai Project Study Group. $N$ Engl J Med 2000;342(13):921-9.

20. Pilcher CD, Price MA, Hoffman IF, et al. Frequent detection of acute primary HIV infection in men in Malawi. Aids 2004;18(3):517-24.

21. Bollinger RC, Brookmeyer RS, Mehendale SM, et al. Risk factors and clinical presentation of acute primary HIV infection in India. Jama 1997;278(23):2085-9.

22. Pilcher CD, Tien HC, Eron JJ, Jr., et al. Brief but efficient: acute HIV infection and the sexual transmission of HIV. J Infect Dis 2004;189(10):1785-92.

23. Rottingen JA, Cameron DW, Garnett GP. A systematic review of the epidemiologic interactions between classic sexually transmitted diseases and HIV: how much really is known? Sex Transm Dis 2001;28(10):579-97.

24. Sexton J, Garnett G, Rottingen JA. Metaanalysis and metaregression in interpreting study variability in the impact of sexually transmitted diseases on susceptibility to HIV infection. Sex Transm Dis 2005;32(6):351-7.

25. Korenromp EL, Sudaryo MK, de Vlas SJ, et al. What proportion of episodes of gonorrhoea and chlamydia becomes symptomatic? Int J STD AIDS 2002;13(2):91-101. 26. Vickerman P, Watts C, Peeling RW, et al. Modelling the cost effectiveness of rapid point of care diagnostic tests for the control of HIV and other sexually transmitted infections among female sex workers. Sex Transm Infect 2006;82(5):403-412.

27. Golden MR, Schillinger JA, Markowitz L, et al. Duration of untreated genital infections with chlamydia trachomatis: a review of the literature. Sex Transm Dis 2000;27(6):329-37.

28. Buve A, Weiss HA, Laga M, et al. The epidemiology of gonorrhoea, chlamydial infection and syphilis in four African cities. Aids 2001;15 Suppl 4:S79-88.

29. Wi T, Ramos ER, Steen R, et al. STI declines among sex workers and clients following outreach, one time presumptive treatment, and regular screening of sex workers in the Philippines. Sex Transm Infect 2006;82(5):386-91.

30. Rou K, Wu Z, Sullivan SG, et al. A five-city trial of a behavioural intervention to reduce sexually transmitted disease/HIV risk among sex workers in China. Aids 2007;21 Suppl 8:S95-101.

31. Wang H, Wang N, Chen RY, et al. Prevalence and predictors of herpes simplex virus type 2 infection among female sex workers in Yunnan Province, China. Int J STD AIDS 2008;19(9):635-9. 
32. Quinn TC, Gaydos C, Shepherd M, et al. Epidemiologic and microbiologic correlates of Chlamydia trachomatis infection in sexual partnerships. Jama 1996;276(21):1737-42.

33. Karmon E, Potts M, Getz WM. Microbicides and HIV: help or hindrance? J Acquir Immune Defic Syndr 2003;34(1):71-5.

34. Smith RJ, Bodine EN, Wilson DP, et al. Evaluating the potential impact of vaginal microbicides to reduce the risk of acquiring HIV in female sex workers. AIDS 2005;19(4):413-21.

35. Alary M, Lowndes CM, Guedeme A, et al. Scaling up prevention interventions for female sex workers in Benin: impact on HIV and STI prevalence. 17th International Society for Sexually Transmitted Diseases Research; 29 July - 1 August 2007; Seattle, Washington, USA [Abstract O-090]. http://www.isstdr.org/index.php?id=97.

36. Lowndes CM, Alary M, Labbe AC, et al. Interventions among male clients of female sex workers in Benin, West Africa: an essential component of targeted HIV preventive interventions. Sex Transm Infect 2007;83(7):577-81.

37. Vu Nguyen T, Van Khuu N, Thi Le TT, et al. Sexually Transmitted Infections and Risk Factors for Gonorrhea and Chlamydia in Female Sex Workers in Soc Trang, Vietnam. Sex Transm Dis 2008.

38. Xu JJ, Wang N, Lu L, et al. HIV and STIs in clients and female sex workers in mining regions of Gejiu City, China. Sex Transm Dis 2008;35(6):558-65.

39. Foss AM, Watts CH, Vickerman PT, et al. The importance of a microbicide's STI-efficacy in reducing HIV-risk and its affect on the reduction in condom use that can be tolerated following product introduction: model projections. 17th International Society for Sexually Transmitted Diseases Research; 29 July - 1 August 2007; Seattle, Washington, USA [Abstract 636]. http://www.isstdr.org/index.php?id=97.

40. Desai K, Boily MC, Garnett GP, et al. The role of sexually transmitted infections in male circumcision effectiveness against HIV--insights from clinical trial simulation. Emerg Themes Epidemiol 2006;3:19.

41. Chen FH. The impact of microbicides and changes in condom usage on HIV prevalence in men and women. AIDS 2006;20(11):1551-1553. 


\section{TABLES AND FIGURES}

Table 1: Input parameters used in the static HIV-dynamic STI revised model for microbicides

\begin{tabular}{|c|c|c|}
\hline Parameter definition & Value & References and notes \\
\hline$\%$ of sex acts in which condom is used before microbicide introduction & $56 \%$ & Condom use at last commercial sex reported by client was $55.8 \% .^{8}$ \\
\hline$\%$ of non-condom-protected sex acts in which microbicide is used & $50 \%$ & Scenario, based on levels of spermicide use observed in trials. ${ }^{6}$ \\
\hline Per sex act HIV-efficacy of condom & $95 \%$ & $\begin{array}{l}\text { Model-based estimate suggests condom use reduces male-to-female transmission of } \\
\text { HIV by } 95 \% \text { per sex act. }{ }^{9}\end{array}$ \\
\hline Per sex act STI-efficacy of condom & $95 \%$ & $\begin{array}{l}\text { Assumed equal to HIV-efficacy of condoms. High-end estimate used here so that } \\
\text { model findings about effect of microbicide are conservative. }\end{array}$ \\
\hline Per sex act HIV-efficacy of microbicide & $50 \%$ & Scenarios \\
\hline Per sex act STI-efficacy of microbicide & $\begin{array}{c}50 \% \\
(0,25 \%, 50 \%, 75 \%)[0-100 \%]\end{array}$ & Scenarios \\
\hline Average number of sexual partnerships per month & $\begin{array}{l}56 \text { clients per FSW }(m) \\
(28,56,112)[20-112] \\
2.7 \text { FSWs per client }(M)\end{array}$ & $\begin{array}{l}\text { FSW s reported a mean of } 13 \text { clients in previous week. }{ }^{10}{ }^{11} \text { Clients reported a mean of } \\
2.7 \text { visits to FSWs per month. }{ }^{8} \text { Assumed any regular/repeat clients visit the same FSW } \\
\text { no more than once per month. }\end{array}$ \\
\hline Average number of sex acts per partnership per month & 1 & Fitted parameter/scenarios \\
\hline Per sex act transmission probability of HIV & 0.002 male-to-female & $\begin{array}{l}\text { Reviews suggest male-to-female transmission probability is } \sim 0.002^{12} \text { (in developing } \\
\text { countries }^{13} \text { ). }\end{array}$ \\
\hline
\end{tabular}




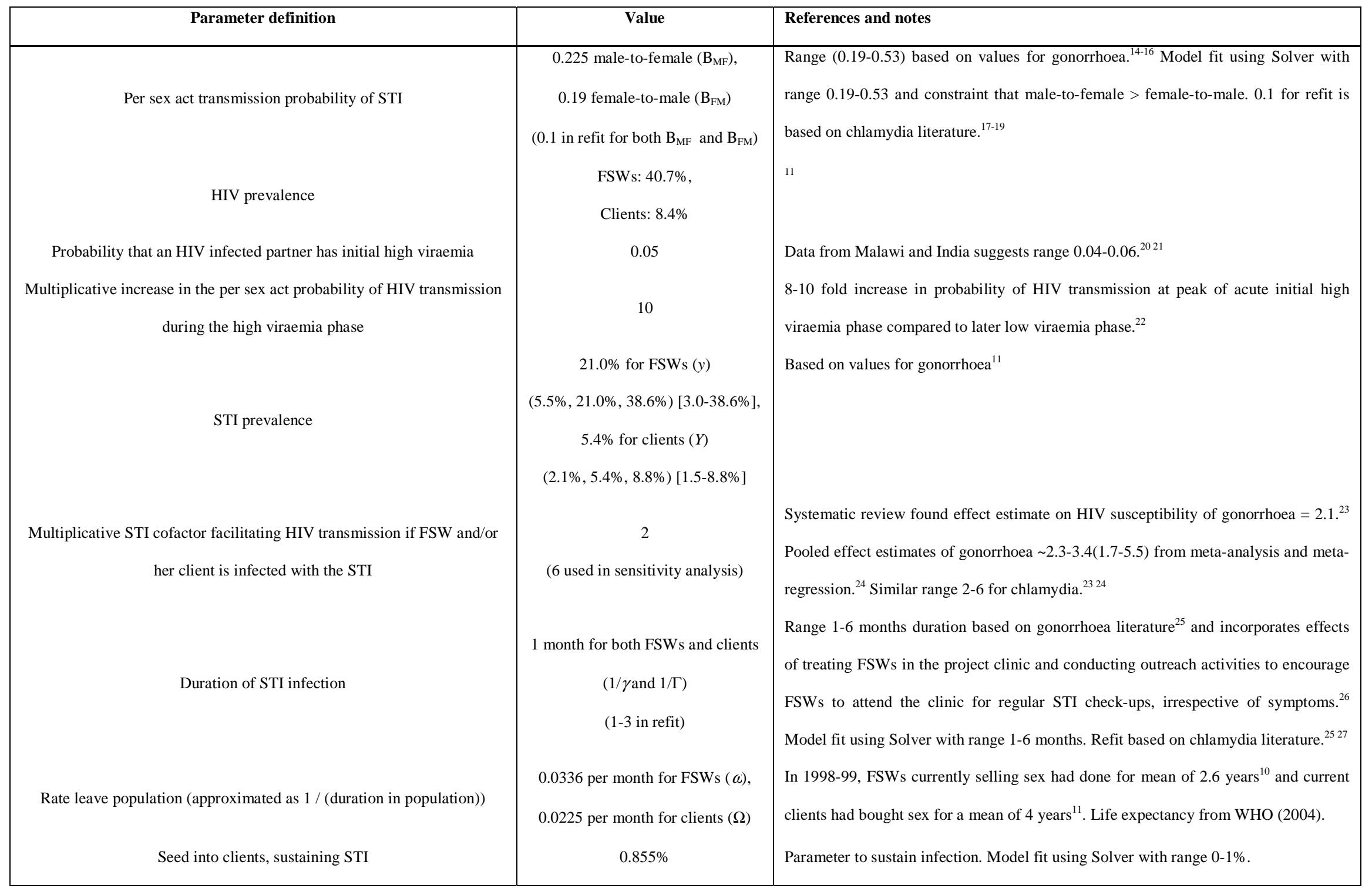


Figure 1: Reduction in HIV-risk among sex workers (from the combined effect of a $50 \%$ HIV-efficacy microbicide with four different STI-efficacies) for different STI prevalences

1a: Original model fit using transmission probabilities of 0.2 per sex act, with different STI prevalences obtained by varying the number of clients per FSW

1b: Additional model refit using transmission probabilities of 0.1 per sex act, with different STI prevalences obtained by varying the duration of the STI 
Figure 2: Reduction in HIV-risk among sex workers (from the combined effect of a 50\% HIV-efficacy microbicide with different STI-efficacies) for three different STI prevalences, assuming a reduction in condom consistency of $16 \%$

2a: Original model fit using transmission probabilities of 0.2 per sex act, with different STI prevalences obtained by varying the number of clients per FSW

2b: Additional model refit using transmission probabilities of 0.1 per sex act, with different STI prevalences obtained by varying the duration of the STI 
Figure 1: Reduction in HIV-risk among sex workers (from the combined effect of a $50 \%$ HIV-efficacy microbicide with four different STI-efficacies) for different STI prevalences

a: Original model fit using transmission probabilities of 0.2 per sex act, with different STI prevalences obtained by varying the number of clients per FSW

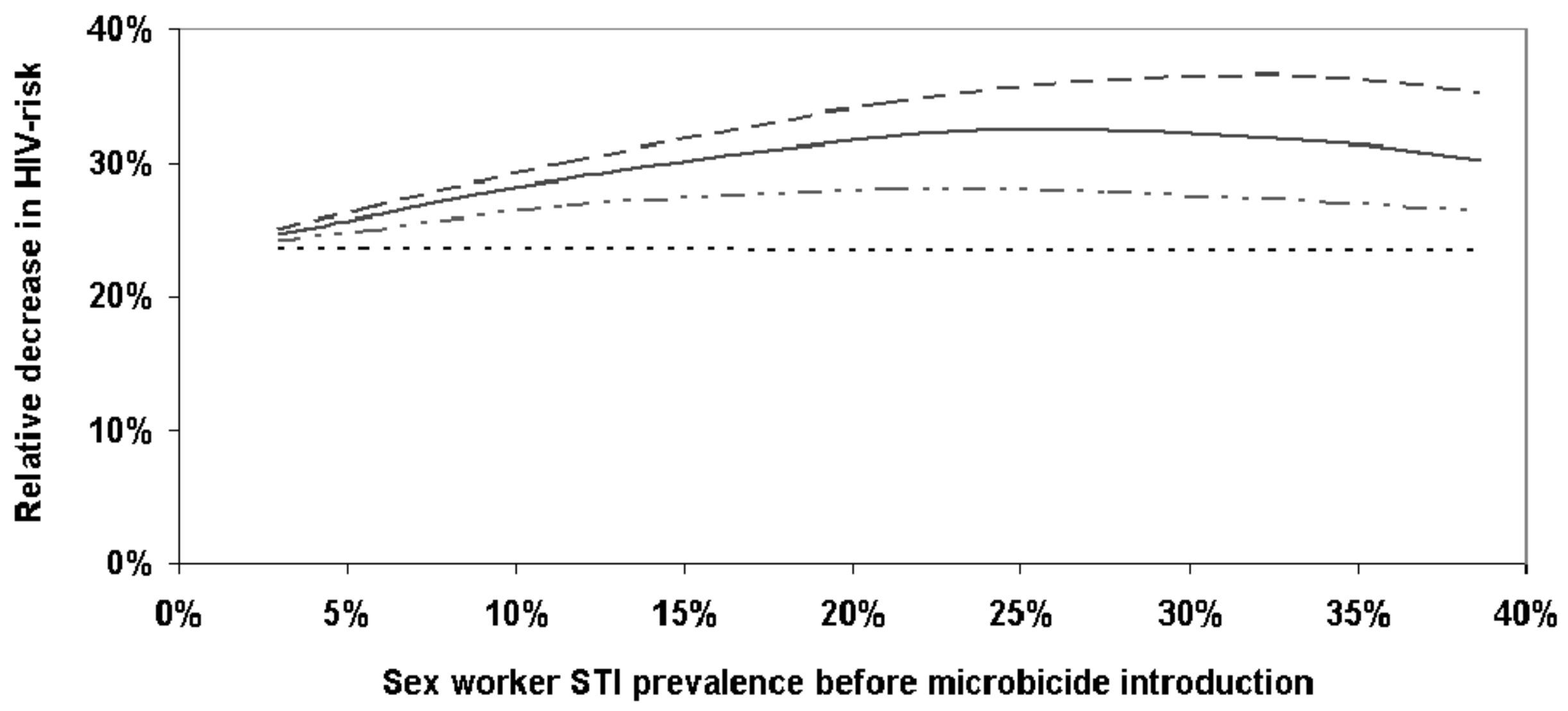

- . . 0 0 STI-efficacy - - - 25\% STI-efficacy —-50\% STI-efficacy - - - $75 \%$ STI-efficacy 
Figure 1: Reduction in HIV-risk among sex workers (from the combined effect of a $50 \%$ HIV-efficacy microbicide with four different STI-efficacies) for different STI prevalences

b: Additional model refit using transmission probabilities of 0.1 per sex act, with different STI prevalences obtained by varying the duration of the STI

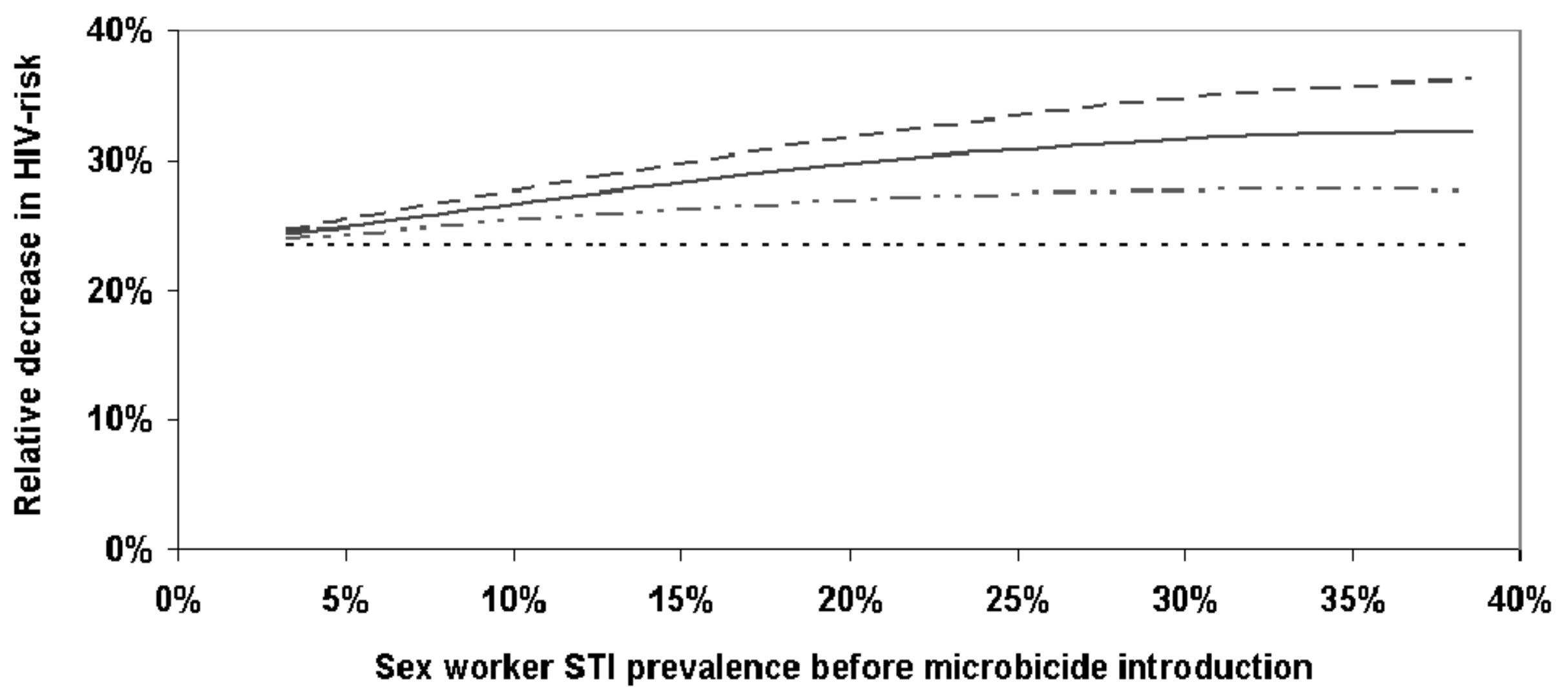

$\cdots+-0 \%$ STI-efficacy $-\cdots 25 \%$ STI-efficacy $-50 \%$ STI-efficacy $---75 \%$ STI-efficacy 
Figure 2: Reduction in HIV-risk among sex workers (from the combined effect of a 50\% HIV-efficacy microbicide with different STI-efficacies) for three different STI prevalences, assuming a reduction in condom consistency of $16 \%$

a: Original model fit using transmission probabilities of 0.2 per sex act, with different STI prevalences obtained by varying the number of clients per FSW

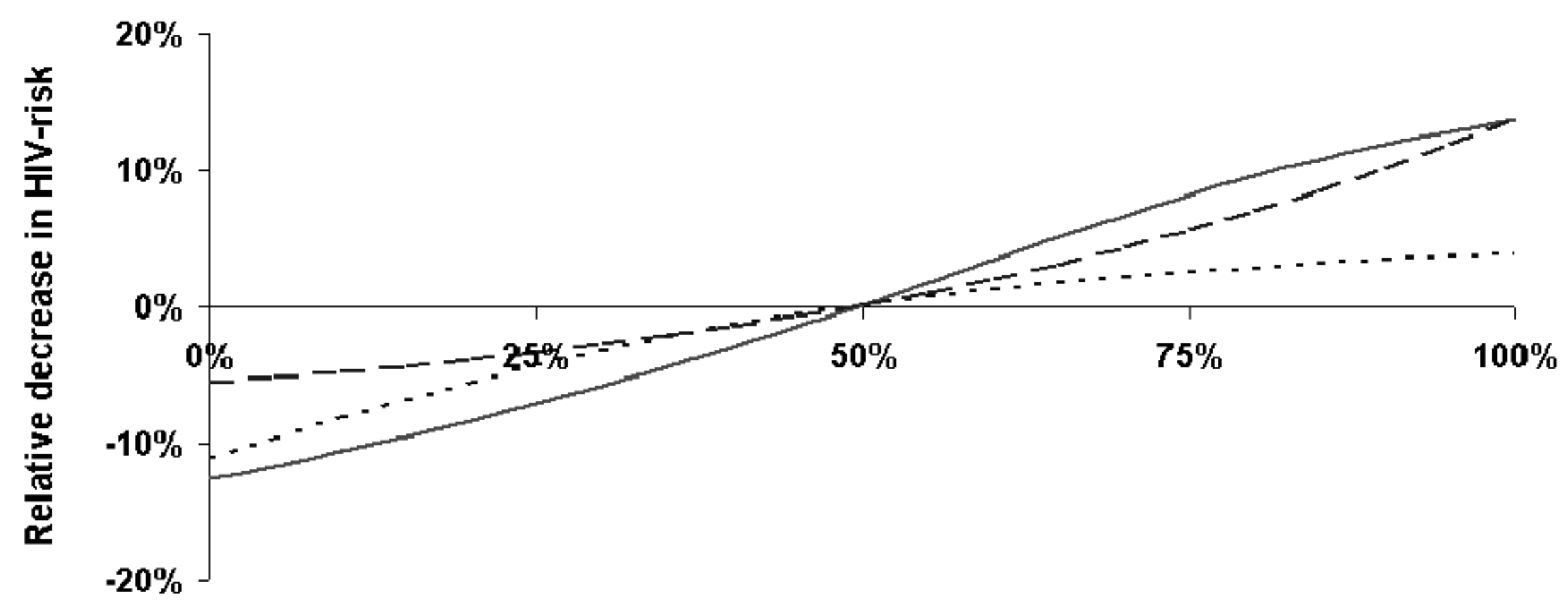

STI-efficacy of microbicide

- . - - Low STI prevalence _ Medium STI prevalence $\quad$ - - High STI prevalence


Figure 2: Reduction in HIV-risk among sex workers (from the combined effect of a $50 \%$ HIV-efficacy microbicide with different STI-efficacies) for three different STI prevalences, assuming a reduction in condom consistency of $16 \%$

b: Additional model refit using transmission probabilities of 0.1 per sex act, with different STI prevalences obtained by varying the duration of the STI

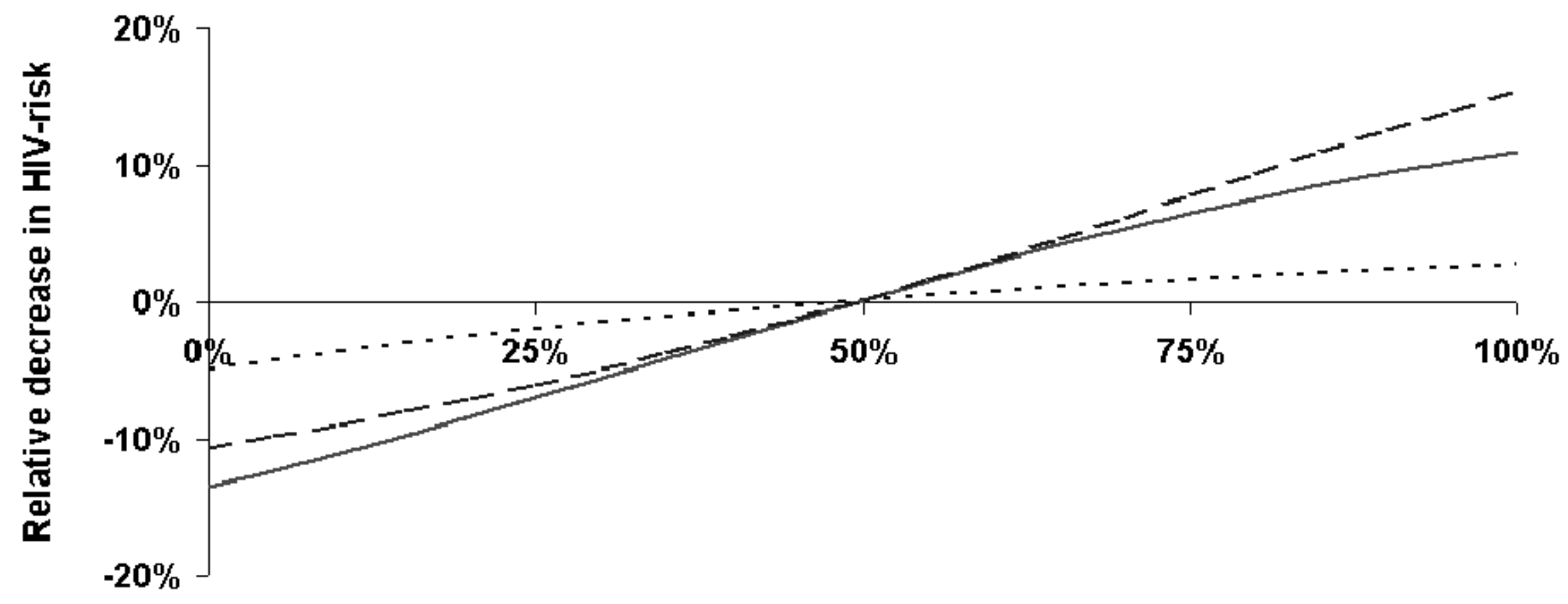

STI-efficacy of microbicide 